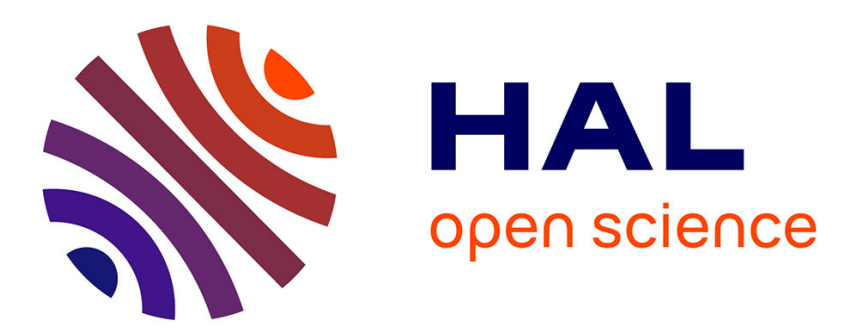

\title{
Interroger la littératie en santé dans une perspective de transformations individuelles et sociales
}

Maryvette Balcou-Debussche

\section{To cite this version:}

Maryvette Balcou-Debussche. Interroger la littératie en santé dans une perspective de transformations individuelles et sociales: Analyse de l'évolution de 42 personnes diabétiques sur trois ans. Recherches \& éducations, 2016, 16, pp.73-86. hal-01697573

\section{HAL Id: hal-01697573 \\ https://hal.univ-reunion.fr/hal-01697573}

Submitted on 31 Jan 2018

HAL is a multi-disciplinary open access archive for the deposit and dissemination of scientific research documents, whether they are published or not. The documents may come from teaching and research institutions in France or abroad, or from public or private research centers.
L'archive ouverte pluridisciplinaire HAL, est destinée au dépôt et à la diffusion de documents scientifiques de niveau recherche, publiés ou non, émanant des établissements d'enseignement et de recherche français ou étrangers, des laboratoires publics ou privés. 


\section{Recherches \& éducations}

16 | Octobre 2016

Émancipation et formation de soi Tome 2

\section{Interroger la littératie en santé dans une perspective de transformations individuelles et sociales}

Analyse de l'évolution de 42 personnes diabétiques sur trois ans

\section{Maryvette Balcou-Debussche}

revues.org

Édition électronique

URL : http://

rechercheseducations.revues.org/3240

ISSN : 1760-7760
Éditeur

Société Binet Simon

Édition imprimée

Date de publication : 1 octobre 2016

Pagination : 73-86

ISSN : 1969-0622

Référence électronique

Maryvette Balcou-Debussche, «Interroger la littératie en santé dans une perspective de

transformations individuelles et sociales », Recherches \& éducations [En ligne], 16 | Octobre 2016, mis

en ligne le 30 juin 2017, consulté le 05 juillet 2017. URL : http://rechercheseducations.revues.org/3240

Ce document a été généré automatiquement le 5 juillet 2017.

Propriété intellectuelle 


\section{Interroger la littératie en santé dans une perspective de transformations individuelles et sociales}

Analyse de l'évolution de 42 personnes diabétiques sur trois ans

Maryvette Balcou-Debussche

\section{Éléments de problématisation}

1 La question de l'émancipation est bien plus complexe qu'il n'y paraît car elle interroge tout autant les acteurs eux-mêmes que les dispositifs mis à leur disposition, les méthodes mobilisées lors des interventions éducatives et des investigations, ainsi que les normes sociales et culturelles différenciées auxquelles chacun se réfère. L'émancipation renvoie à des considérations éthiques fondamentales, notamment quand l'intervention concerne des personnes adultes malades chroniques engagées dans un processus éducatif et de soins en structure hospitalière. Les modifications des rapports aux savoirs et à la maladie invitent à l'envisager sous plusieurs formes allant de la prise de distance par rapport à la maladie elle-même ou aux contraintes des environnements en présence, jusqu'à la renégociation des relations avec le corps médical qui encadre et suit l'évolution de la maladie. Ici, nous interrogeons les résultats obtenus par 42 patients vivant à La Réunion, inclus dans la recherche longitudinale ERMIès-ethnosocio qui visait à analyser de façon fine les processus d'apprentissage, d'adaptation, d'autonomisation et d'appropriation des connaissances de personnes diabétiques dites "insuffisamment équilibrées", ainsi que leur évolution sur deux années. Les analyses portent sur les résultats en matière de littératie en santé, les contours théoriques et méthodologiques étant pensés à travers deux facettes ambivalentes des processus émancipatoires chez les personnes malades chroniques : à la fois la volonté et/ou la nécessité de s'affranchir de la dépendance du monde médical et en même temps, le besoin impératif ou le désir de la conserver (Thorne et al. 2000 ; Fainzang, 2006 ; Bureau et Hermann-Mesfen, 2014). 
Ce questionnement s'inscrit dans le cadre d'interventions éducatives et sociales envisagées comme construit théorique de la praxis (Lenoir et al., 2002) où se jouent un certain nombre de constructions et de déconstructions qu'il s'agit de lire à la lueur des contextes mobilisés. Au cours de toute intervention, chaque groupe, chaque personne peut faire preuve d'adhésion, d'opposition, de mécanismes de défense ou d'abandon, notamment lorsque l'intervention se réalise selon des caractéristiques répressives. L'approche privilégiée ici consiste à faire en sorte que l'individu soit placé dans des conditions d'apprentissage appropriées permettant qu'il soit pris en considération et reconnu comme responsable, ce qui relève d'une option sociale fondée sur le souci d'une émancipation de l'être humain en perspective de développements démocratiques tels que les a définis John Dewey (1939). Ici, nous étudions en quoi une démarche anthropologique d'éducation peut participer aux développements différenciés des individus (Benoist, 2003 ; Fainzang, 2005 ; Balcou-Debussche, 2012, 2014, 2016). Le processus de l'expérience partagée importe alors davantage que tel ou tel résultat particulier et le processus de démocratie créatrice n'est pas coupé de l'esprit scientifique puisqu'il valorise l'enquête, l'expérimentation, la transformation de la théorie et des idées au contact des faits nouveaux (Leroux, 1995 ; Stavo-Debauge et Trom, 2004). Enfermer les individus dans les limites de leur culture ou de leur origine sociale reviendrait à empêcher toute possibilité d'émancipation, de progression ou de déplacement social (Lahire, 2013) : les socialisations des individus et leurs possibilités d'être singuliers sont donc envisagées de concert.

Ce questionnement s'inscrit aussi dans un contexte général de forte prévalence du diabète de type 2 dans le monde (proche de $8 \%$ dans la population adulte et plus de 15 à $20 \%$ dans plusieurs pays). ${ }^{1}$ Parallèlement aux soins, l'efficacité de l'éducation thérapeutique dans les maladies chroniques est unanimement reconnue, même si les modalités en restent encore débattues (Debussche, 2015). La focalisation sur des changements de comportements en vue d'améliorer les indicateurs biomédicaux aboutit plus souvent à la négociation de responsabilités qu'à une analyse réflexive partagée (Thille et al., 2014). Pourtant, les personnes malades chroniques sont capables de développer des compétences dans une perspective de santé, d'émancipation et de formation de soi, en interrelation étroite avec les environnements concernés (BalcouDebussche, 2014, 2016). Développer la littératie en santé conduit alors à penser la complexité et la diversité des individus à travers une connaissance d'usages différenciés du langage (Rispail, 2011; Auriac-Slusarczyk, 2013) et à interroger les possibilités de mobiliser les usages sociaux relatifs au monde de la santé (Osborne et al., 2013). Cette perspective se nourrit notamment des travaux scientifiques sur les appropriations de savoirs (Bautier et Goigoux, 2004), la construction sociale de l'illettrisme (Lahire, 2005) et le rôle du contexte dans les apprentissages (Bril, 2002). De par les transformations cognitives et sociales qu'il engendre, un travail sur la littératie est susceptible de modifier les rapports de pouvoir entre les protagonistes de la relation pédagogique ou didactique, dont le formateur (le soignant) et l'apprenant (le patient). Le projet exige d'analyser un certain nombre de résultats avant, pendant et après l'intervention éducative, ce qui permet d'expliciter l'expérience selon une visée à la fois réflexive et critique qui souligne que le processus a permis ou non des émancipations entendues comme un bénéfice pour les individus concernés. Ce bénéfice se traduit a priori par la possibilité de poursuivre son propre cheminement en ayant une meilleure compréhension des enjeux liés à la maladie chronique et à sa gestion, et en ayant une capacité affirmée à réaliser des choix, à prendre des décisions et à « circuler » dans des contextes mouvants qui exigent des adaptations individuelles et collectives permanentes. 


\section{Deux corpus complémentaires}

2 La recherche qualitative ERMIèS-ethnosocio est intégrée à la recherche épidémiologique ERMIès dont l'objectif est de tester l'efficacité d'une intervention éducative maintenue sur 2 ans auprès de personnes présentant un diabète insuffisamment équilibré. Le recueil de données a été effectué en deux temps :

Phase 1 / Corpus 1 : Parmi les 102 patients réunionnais inclus dans la recherche ERMIès, nous avons interrogé 44 personnes volontaires, à leur domicile, en 2012, après l'inclusion. Ces 44 patients ont intégré la recherche qualitative en ayant été sollicités «au fil de l'eau ». D'une durée totale de 20 à $40 \mathrm{mn}$, les entretiens semi-directifs ont porté sur le diabète et le risque cardiovasculaire, l'auto-surveillance glycémique, l'accès aux informations en santé, les rapports à l'éducation et à l'apprentissage, le positionnement du malade et ses prises de décisions, le regard critique sur l'environnement du malade chronique.

Phase 2 / Corpus 2 : Sur les 44 patients, 42 ont été revus en 2015. Nous avons utilisé une grille de recueil de données qui a été élaborée à partir de l'analyse des 44 entretiens de 2012 et de 12 entretiens exploratoires menés en 2013. ${ }^{2}$ Dans une perspective longitudinale, nous avons mis en relation les données relatives aux différents " niveaux » de littératie en santé avec les parcours de soins des 42 patients.

Les 42 patients, nés et vivant à la Réunion, se répartissaient ainsi : 30 femmes, 12 hommes. Sur les 42, la moitié (21 personnes) ont plus de 60 ans. 12 sont sans activité professionnelle, 18 sont retraités et 9 sont employés (un seul artisan et deux professions intermédiaires). 13 personnes ont un niveau de revenu du foyer supérieur à 1200 euros par mois. Du côté du parcours éducatif, les personnes se répartissent ainsi: pas de scolarité (3), niveau primaire (6), collège (23), lycée (5), supérieur (5).

\section{Trois rapports différenciés à la littératie en santé}

3 Les entretiens ont fait l'objet d'une analyse de discours, entendus non pas comme des types de textes, mais comme des dispositifs de communication de nature à la fois sociale et linguistique (Maingueneau, 2012). Les catégories et les signifiants ont été développés en utilisant la méthode de comparaison constante (Glaser et Strauss, 1967). Le travail a été réalisé sur le logiciel d'analyse qualitative N-Vivo 10 QSR International.

L'analyse des 44 entretiens réalisés en 2012 a permis de déterminer huit variables constitutives de la gestion du diabète en contexte ordinaire. En prenant appui sur les catégories établies par Don Nutbeam en littératie en santé (Nutbeam, 2008), trois types de rapports ont été déterminés :

- Un « rapport fonctionnel à ... », soit un rapport instrumental à (l'alimentation, l'activité physique...) en lien avec des tâches exercées sans regard critique dans des situations quotidiennes.

- Un « rapport interactif à... » qui conduit les patients à mobiliser des compétences cognitives et sociales pour participer activement aux activités de tous les jours, déduire le sens de différentes formes de communication et appliquer les informations nouvelles à des circonstances changeantes. 
- Un « rapport critique à... » avec des compétences cognitives et sociales plus avancées, appliquées à une analyse critique des informations et à leur utilisation pour exercer un plus grand contrôle sur les événements de la vie et les situations.

4 L'analyse des données a permis d'attribuer à chaque personne une première "étoile 2012 » telle que nous la présentons ci-dessous. Les différents "rapports à » ont été identifiés pour chacune des variables, et pour chaque personne. A partir de ces premières analyses, nous avons construit une grille méthodologique qualitative structurée autour des 8 variables et des trois « niveaux » fonctionnel, interactif et critique. Cette grille a été proposée aux 42 patients revus en 2015. L'assistante de recherche remplissait la grille en fonction des propos tenus en situation, mais la grille était aussi remplie par le patient. Cette double validation a permis de construire les 42 "étoiles 2015 », avec la mise en relation des résultats de 2012 avec ceux de 2015. Ce sont les résultats de cette évolution que nous présentons ci-après, la perspective étant d'analyser les indicateurs qui rendent compte d'une émancipation des personnes pour l'ensemble des variables, ou pour certaines d'entre elles.

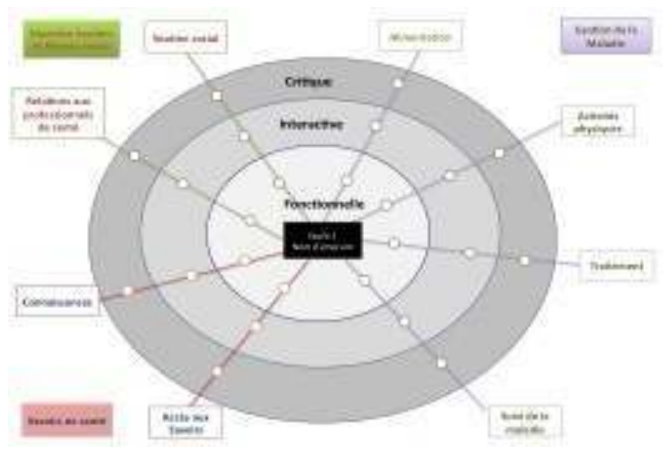

Figure 1 : étoile mettant en évidence les 8 variables et les 3 « niveaux »

\section{COMPLETER SCHEMA}

6 Le «niveau » fonctionnel baisse pour les trois variables, au profit de l'interactif et du critique qui progressent (sauf le critique qui baisse d'un point, pour l'alimentation). Ces résultats soulignent l'impact d'un processus de soins structuré et intensifié, auquel s'est ajouté en parallèle un processus éducatif composé de plusieurs situations d'apprentissage complémentaires (Balcou-Debussche, 2012). ${ }^{3}$ S'agissant de l'alimentation, l'évolution entre les différents " niveaux » se traduit à travers des propos tels qu'ils sont présentés dans le tableau ${ }^{4}$ ci-dessous :

Tableau 1 : Exemples de variations des discours selon les « niveaux » fonctionnel, interactif et critique

\begin{tabular}{|l|l|l|}
\hline Fonctionnel & $\begin{array}{l}\text { Delphine, } \\
50 \text { ans, } \\
\text { sans } \\
\text { activité }\end{array}$ & $\begin{array}{l}\text { Pour manger, je mange à peu près comme l'hôpital nous dit de le } \\
\text { faire et ce qu'il nous donne. }\end{array}$ \\
\hline Interactif & $\begin{array}{l}\text { Ludivine, } \\
76 \quad \text { ans, } \\
\text { retraitée }\end{array}$ & $\begin{array}{l}\text { J'arrive même quand je fais une bêtise au cours d'un repas, à corriger } \\
\text { la fois d'après. Je sais comment suivre mon alimentation et comment } \\
\text { la faire valoir par rapport à mon diabète. }\end{array}$ \\
\hline
\end{tabular}




\begin{tabular}{|l|l|l|}
\hline Critique & $\begin{array}{l}\text { Martin, } \\
75 \\
\text { retraité }\end{array}$ & $\begin{array}{l}\text { La nourriture comment ça marche ? Ça va. Je ne mange pas trop de } \\
\text { sucre, pas trop de sel. Je ne bois pas trop d'alcool. C'est bon, je mange } \\
\text { de tout, je mange bien, j'ai un bon appétit. }\end{array}$ \\
\hline
\end{tabular}

7 En 2015, un nombre important de patients a compris qu'une alimentation équilibrée est une alimentation variée, en quantités adaptées, la notion d'interdits étant reléguée au profit des idées de modération et d'exceptions (lors des fêtes de famille, par exemple). Ils ont compris les bienfaits de l'activité physique en lien direct avec le diabète. Dans bon nombre de cas, l'activité physique devient un véritable plaisir qui s'intègre aux pratiques ordinaires, procurant ainsi d'autres bénéfices (bien-être, rapports différents au corps, nouvelles dynamiques sociales, emplois du temps bien remplis). Les propos de Ludivine, 76 ans, retraitée, en rendent compte : « Lundi je fais de la gym douce, mardi je fais du taïchi, jeudi je fais de la gym douce, vendredi je fais de la marche dans l'eau. Je suis très active ». Quant au soutien social, les résultats montrent qu'en règle générale, les patients ne se sentent pas isolés dans la gestion de leur maladie. En 2015, 28 patients sur 42 reçoivent un réel soutien familial ou amical qui se traduit tout autant par la solidarité que le partage au sein de la famille. Ce tissu relationnel se cristallise autour des pratiques alimentaires «Tout le monde mange pareil depuis que je suis diabétique» (Adeline, 73 ans, retraitée), de l'activité physique "Dès fois quand on est décidé, soit c'est elle, soit c'est moi, on fait un petit peu de marche» (Sylvain, 47 ans, artisan) ou dans la compréhension de la maladie : «C'est vrai que mon mari, étant dans le milieu hospitalier, ça m'a beaucoup aidé » (Blandine, 60 ans, employée). Cette solidarité se manifeste différemment en fonction des membres de la famille, ainsi qu'en témoigne Clarisse (55 ans, sans activité professionnelle) : «Oui. Enfin peut-être pas avec mon concubin mais mes enfants, et puis il y a ma famille à côté, mes sœurs, mes frères, mes parents, donc pas de soucis ». Dans certains cas, la personne concernée par le diabète préfère épargner sa famille en assumant elle-même la gestion de la maladie «Pour moi ce n'est pas un handicap, le diabète est là, on gère et point. [...]. Je n'en parle jamais (du diabète), c'est mon affaire » (Sabine, 69 ans, retraitée).

\section{Accès aux savoirs et connaissances}

8 Les résultats sont similaires pour l'accès aux savoirs et le développement des connaissances. Le graphique ci-dessous montre de nouveau que le «niveau » fonctionnel baisse de 2012 à 2015, au profit de l'interactif et du critique : 
Figure 3 : Répartition pour l'accès aux savoirs et les connaissances

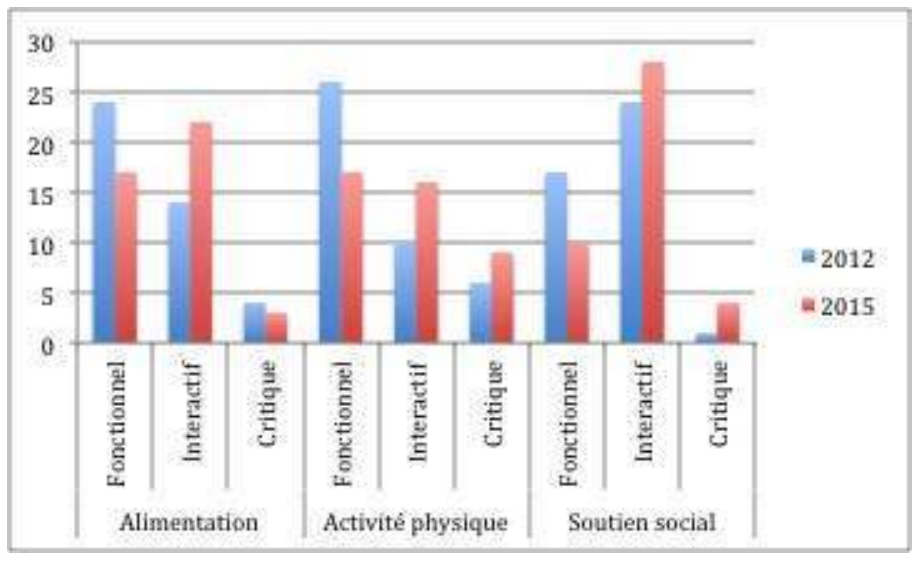

9 Les connaissances sur le diabète varient de la simple explication liée au sucre, telle que Sylvain (46 ans, artisan) l'expose: «Le diabète c'est trop de sucre dans le sang ». Au «niveau » interactif, les patients sont capables d'établir des liens entre au moins deux composantes, comme par exemple les liens entre le diabète et une alimentation équilibrée, ou la pratique d'une activité physique régulière. Au "niveau » critique, les patients évoquent un système complexe, dans lequel les interactions entre les différents éléments constitutifs participent à l'évolution de la maladie et de ses complications : «Le diabète c'est une maladie silencieuse qui attaque tous les organes vitaux comme le cœur, les reins. Ça peut arriver jusqu'à couper des membres. On sait que c'est une maladie très grave » (Sébastien, 48 ans, employé).

La présence des nouvelles technologies et d'internet dans un grand nombre de foyers a considérablement modifié les rapports aux savoirs, ce qui n'empêche pas les variations selon les patients. Certains d'entre eux refusent l'apport de nouvelles informations par crainte de découvrir, à l'avance, les incidences de la maladie: «Je ne regarde pas trop internet parce que tout ce qui est médical me fait peur. Donc en général, quand j'ai besoin d'une information, je téléphone à une diététicienne de l'hôpital " (Mélanie, 49 ans, profession intermédiaire). Parfois, les patients estiment qu'ils en savent assez sur leur maladie et sa gestion : " On écoute la radio, on est allé à des réunions sur comment faire à manger. Maintenant on est un peu fatigué d'y aller parce qu'on nous répète des choses que l'on sait déjà » (Martin, 75 ans, retraité). D'autres souhaitent avoir davantage d'informations, ce qui leur donne la possibilité de comparer les données et/ou de suivre l'actualité, dont par exemple les évolutions des médicaments : "Hier je suis allé chez le docteur et tout est bon. Mais mon corps ne va pas bien du tout [...] ils ne veulent pas entendre (les médecins) que c'est le médicament. Je suis allé voir sur internet les médicaments que je prends, et pour la plupart les symptômes que j'ai correspondent » (Marc, 48 ans, sans activité professionnelle).

Tableau 2 : Exemples de variations (fonctionnel, interactif, critique) pour l'accès aux savoirs

\begin{tabular}{|l|l|l|}
\hline Fonctionnel & $\begin{array}{l}\text { Irène, } \\
\text { ans, } \\
\text { retraitée }\end{array}$ & $\begin{array}{l}\text { J'essaie d'écouter (la radio ou la télé), j'essaie de comprendre, mais } \\
\text { ça rentre dans ma tête, mais je ne comprends pas, moi. }\end{array}$ \\
\hline
\end{tabular}




\begin{tabular}{|l|l|l|}
\hline \multirow{3}{*}{ Interactif } & $\begin{array}{l}\text { Constance, } \\
64 \quad \text { ans, } \\
\text { retraitée }\end{array}$ & $\begin{array}{l}\text { Les articles de journaux, tous les mois, il y a un médicament } \\
\text { pharma, pour femme, donc je prends ça à la pharmacie. Dès fois, si } \\
\text { ma fille arrive avec son ordinateur, elle cherche, je demande et } \\
\text { puis je reçois Sophia, on m'envoie des documents tout le temps, } \\
\text { donc je lis, il y a des témoignages, il y a des médicaments, comment } \\
\text { on fait, comment ne pas faire. Voilà donc je suis tout ça de très } \\
\text { près. }\end{array}$ \\
\hline Critique & $\begin{array}{l}\text { Damien, } \\
73 \\
\text { retraité }\end{array}$ & $\begin{array}{l}\text { Le diabète, au niveau du prix, des médicaments, c'est lié à des } \\
\text { recherches, à des laboratoires qui mettent aussi ce qu'ils veulent, et } \\
\text { puis il y a des farfelus qui peuvent écrire n'importe quoi. Donc de } \\
\text { ce côté là, il y a un manque de contrôle de ce que l'on montre sur } \\
\text { internet. [...] C'est une mine d'or, mais il faut savoir si c'est de l'or, } \\
\text { du vrai ou du synthétique. }\end{array}$ \\
\hline
\end{tabular}

\section{Traitement, suivi de maladie et relations avec les soignants}

10 Un même mouvement s'observe entre 2012 et 2015 pour les variables traitement, suivi de la maladie et relations avec les soignants. De nouveau, le «niveau » fonctionnel baisse alors que l'interactif et le critique progressent.

\section{Figure 4 : Répartition pour le traitement, le suivi de la maladie et les relations avec les soignants}

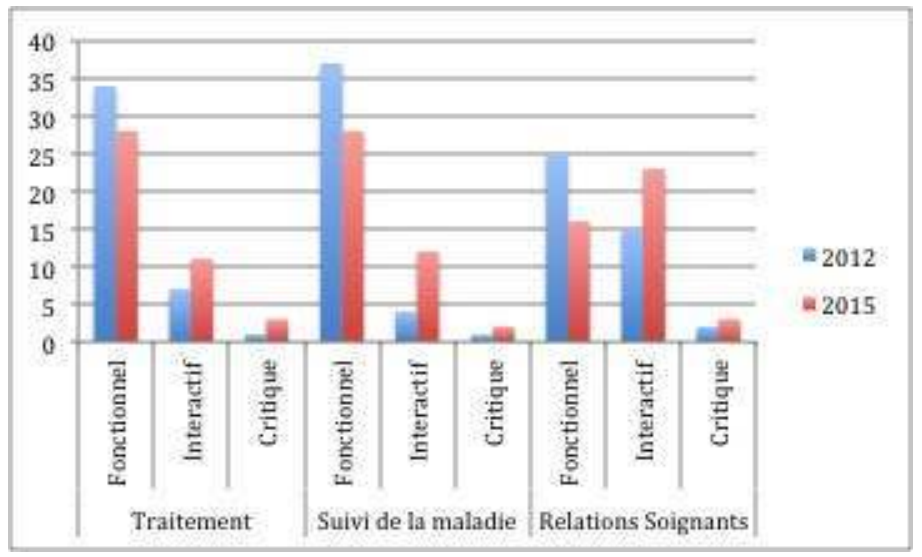

11 Ce qui diffère néanmoins, c'est la distribution des personnes dans les différents « niveaux », et ce, dès 2012. Sur 42 patients, 34 d'entre eux sont au « niveau » fonctionnel pour le traitement, 36 le sont pour le suivi de la maladie et 25 au niveau des relations avec les soignants. A titre de comparaison, en 2012, ils ne sont que 17 au « niveau » fonctionnel pour le soutien social. On observe ainsi que, pour ces trois variables, la question de l'émancipation se pose dans d'autres termes. En 2015, la plupart des patients appliquent encore les prescriptions à la lettre sans véritablement en comprendre le sens : "On me conseille, on me donne un traitement, je suis à la lettre et puis point barre » (Solange, 43 ans, employée). Seuls quelques-uns d'entre eux procèdent à des ajustements eux-mêmes, ainsi qu'en rend compte Olivia, 60 ans, sans activité professionnelle: "Par exemple j'augmente les doses d'insuline quand c'est trop fort, et le repas aussi, j'évite de manger 
un peu plus, j'essaye de diminuer les portions ». Parmi les rares patients qui se situent au « niveau » critique en 2015, Guillaume, 69 ans, retraité, précise ceci : « Les injections, j'en ai deux le matin et une le soir et c'est pratiquement aussi automatique que de se brosser les dents. Ça fait dix ans que j'ai les injections, j'ai dû oublier deux fois parce que j'étais en voyage ou les circonstances qui font que c'est complètement automatisé ».

S'agissant du suivi de la maladie et des relations avec les soignants, on retrouve le même type de subordination fonctionnelle pour la majorité des patients. Clarisse, 55 ans, sans activité professionnelle précise ceci : « Normalement c'était trois fois par jour pour voir. Et après on m'a mis deux fois, le matin et le soir. Donc je pique mon doigt et puis voilà ». Irène, 69 ans, retraitée, opère une différenciation entre le médecin qui dit ce qu'il faut faire et l'infirmière qui explique : «J'écoute toujours le docteur, tout ce qu'il me dit. J'ai l'infirmière qui m'explique des choses ». Rares sont les personnes qui, comme Mélanie (52 ans, profession intermédiaire) osent arrêter un traitement et le dire à leur médecin traitant : « Dernièrement j'ai arrêté un des deux médicaments du matin [...]. C'est moi qui en ai parlé (au médecin) ». Plus rares encore sont les patients qui, comme Guillaume (69 ans, retraité), ont capitalisé un certain nombre d'expériences où s'est exercée une réflexivité constructive qui leur permet désormais de gérer au mieux leur diabète en toutes circonstances: «Je vais très rarement chez le docteur ». Pour autant, être au «niveau » critique ne signifie pas que le patient s'affranchit totalement de la relation avec le soignant, car ce qui le caractérise, c'est qu'il est en mesure de savoir à quels moments précis l'intervention du professionnel de santé va être pertinente, nécessaire et non superflue: «Si j'ai un souci je vais voir le docteur B. qui me connaît bien" (Guillaume, 69 ans, retraité).

\section{Discussion et perspectives}

12 Les résultats présentés ici permettent de mettre en évidence les visages pluriels de l'émancipation dès lors qu'il s'agit d'analyser les pratiques ordinaires liées à la gestion du diabète de type 2 chez des personnes qui ont bénéficié d'interventions structurées durant deux années, tant sur le plan médical que sur le plan éducatif. Si le travail de formation de soi est effectif, il ne s'actualise pas de la même façon chez tous les patients, ni selon les variables que l'on considère. L'évolution est bien plus conséquente pour les variables qui concernent le domaine familier du patient (l'alimentation, l'activité physique, le soutien social) alors qu'elle l'est beaucoup moins pour tout ce qui concerne les liens avec le monde de la santé (traitement, suivi de la maladie, relations aux soignants). L'accès aux savoirs et les connaissances constituent un domaine charnière, qui se situe à la croisée des sphères familiale et de la santé puisque l'obtention d'informations relatives à la santé peut se réaliser par le vecteur du monde médical, par l'environnement de proximité ou par les médias et les nouvelles technologies.

L'émancipation des individus est donc à envisager non pas comme une fin en soi, mais à travers les conditions sociales qui autorisent la construction de nouvelles cohérences qui combinent des découvertes (savoirs, expériences) avec la diversité des pratiques sociales de référence et les contextes mobilisés. Cette émancipation mérite d'être pensée à travers une temporalité qui permet de rendre compte d'évolutions suffisamment complexes pour qu'il soit possible d'y distinguer des variations qui seront alors analysées comme les différents répertoires joués par les individus. Dans ces conditions, l'émancipation des personnes est pensée selon une éthique de l'éducation qui envisage que les résultats 
soient différenciés, qu'ils ne concernent pas nécessairement les mêmes variables pour tous et qu'enfin, ces résultats soient rapportés aux contextes dans lesquels les individus négocient et réinterprètent en permanence leurs pratiques sociales. L'émancipation individuelle peut alors se retrouver en adéquation avec une demande sociale diversifiée. Les objets d'enseignement sont réinterprétés pour faire place à des passages, des transitions et des médiations (Lenoir et al. 2002) qui permettent aux individus de s'adapter progressivement à de nouvelles situations et d'apprendre à circuler entre des savoirs issus de différentes sources, entre les experts qui les relaient et les lieux où ces savoirs sont travaillés. L'enjeu est, non pas d'installer à tout prix et à toute heure le malade dans sa maladie, mais de lui permettre de poursuivre le parcours qui est le sien tout en l'amenant à intégrer de façon constructive les nouvelles données relatives à la maladie dont il est porteur, à un rythme qu'il va devoir trouver et selon des déclinaisons qu'il va aussi choisir de renforcer, occulter ou seulement maintenir.

Ainsi, dans le diabète de type 2, les rapports différenciés à la littératie en santé se réfèrent à la capacité des personnes à mettre en œuvre des pratiques pertinentes eu égard à leur état de santé, à en comprendre le sens et à adapter ces pratiques en tenant compte des atouts et des contraintes des contextes dans lesquels elles s'actualisent. Ces capacités, mouvantes, dynamiques et plurielles s'articulent autour de trois pôles: la gestion de la maladie (alimentation, activité physique, traitement, suivi de la maladie), les savoirs en santé (connaissances, accès aux savoirs), l'expertise et le soutien social (soignants, famille). L'articulation cohérente de ces trois pôles et la maîtrise des différentes variables qui les structurent est constitutive d'un maintien de la santé et d'un évitement des complications liées à la maladie chronique. Permettre aux personnes malades de s'émanciper consiste alors à leur offrir les conditions pour qu'elles puissent comprendre et analyser les principaux enjeux liés à leur maladie, tout en apprenant à adapter leurs pratiques ordinaires selon les environnements dans lesquels ces pratiques s'actualisent.

Si l'on s'accorde à dire qu'un projet émancipateur est loin d'une éducation dogmatique uniforme, ou d'une seule diffusion de conseils standardisés, il est important de souligner aussi qu'il ne peut pas être pensé indépendamment d'un mouvement du côté des professionnels de santé. L'émancipation des patients ne peut pas se faire sans une prise de conscience conjointe, chez les soignants, des conditions et conséquences de telles mutations. Nous avons vu ici que les variables qui concernent les liens avec les soignants sont celles qui progressent le moins, même si les résultats montrent des évolutions sur les deux années d'inclusion dans la recherche. Autrement dit, l'émancipation individuelle ne peut pas s'exercer pleinement si elle ne s'accompagne pas d'un mouvement social qui va dans le même sens et qui va permettre de la soutenir et la fortifier.

Actuellement, nous utilisons les analyses issues de cette recherche dans deux directions : à la fois du côté des patients à qui nous restituons les résultats, mais aussi du côté des soignants que nous formons à la prise en compte de ces mutations en cours, avec d'un côté une relative rigidité des structures et des organisations fondées sur des modèles curatifs qui ne répondent plus à tous les besoins des personnes et des sociétés (RothierBautzer, 2002), de l'autre, un mouvement en faveur de codécisions et d'auto-soins, l'activité du patient «contemporain» étant alors sollicitée autour du partage et de la recherche d'autonomie (Fainzang, 2006; Debussche, 2015). Si l'appropriation par le patient remplace progressivement l'idée de transmission à des personnes pensées comme vierges de toute connaissance (Bachelard, 1934), les difficultés persistent néanmoins au niveau de l'accompagnement des personnes vulnérabilisées. Les personnes ayant un faible «niveau » de littératie restent souvent difficiles à associer au travail éducatif, à la 
prise des traitements prescrits et à l'autogestion de la maladie (Greenhalgh, 2015; Nutbeam, 2000). Dans un contexte de développement rapide du nombre de malades chroniques à La Réunion, en France, mais aussi dans le monde (IDF, 2013, 2014), ${ }^{56}$ les travaux sur la littératie en santé et les conditions de son développement, en équipes pluridisciplinaires, devraient ainsi faire l'objet d'une attention grandissante.

\section{Conclusion}

13 Les analyses développées ici permettent de mesurer l'ampleur du chantier qui est encore à développer pour comprendre l'évolution de la « prise en charge » médicale et éducative des malades chroniques, y compris en lien étroit avec l'analyse des interventions éducatives et de soins qui s'opèrent le plus souvent sur la base de dissymétries conséquentes. Permettre des émancipations différenciées, adaptées et contextualisées exige ainsi que soient pensées les voies à travers lesquelles les dispositifs proposés aux patients pourraient offrir un espace bienveillant, capable de permettre de nouvelles élaborations cognitives et sociales, tout en questionnant les soignants dans un même mouvement. S'interroger sur ces conditions conduit dès lors à ne plus envisager l'intervention éducative dans le soin comme un lieu où vont être comblés des manques potentiels, mais bien comme un espace transitionnel où vont pouvoir se construire de nouvelles cohérences entre les savoirs construits et les contextes dans lesquels ils sont supposés s'actualiser. L'heure n'est plus aux querelles de méthodes puisqu'il s'agit d'identifier selon des grains de plus en plus fins les éléments constitutifs qui participent à des évolutions individuelles et collectives structurantes pour les patients, en relation étroite avec le monde des soignants de qui ils ne peuvent s'affranchir totalement. De ce point de vue, le cadre théorique de l'intervention éducative et sociale semble pertinent, y compris en le conjuguant avec les déclinaisons conceptuelles et méthodologiques issues des travaux en plein essor autour de la littératie en santé

\section{BIBLIOGRAPHIE}

Auriac-Slusarczyk, E. (Ed) (2013). Apprendre et former : la dimension langagière. Clermont-Ferrand : Presses Universitaires Blaise Pascal.

Bachelard, G. (1934). La formation de l'esprit scientifique. Contribution à une psychanalyse de la connaissance objective. Paris : Librairie philosophique J. Vrin, 5e édition, 1967.

Balcou-Debussche, M. (2016). De l'éducation thérapeutique à la littératie en santé. Problématisation socio-anthropologique d'objets didactiques contextualisés. Paris : Editions des archives contemporaines.

Balcou-Debussche, M. (2014). Littératie en santé et interactions langagières en éducation thérapeutique. Analyse de situations d'apprentissage au Mali, à La Réunion et à Mayotte. Education, Santé, Sociétés, 1(1), 3-18. 
Balcou-Debussche, M. (2012). L'éducation thérapeutique : entre savoirs complexes, formateurs, apprenants hétérogènes et contextes pluriels. Recherche en Soins Infirmiers, 110, 45-59.

Bautier, E. \& Goigoux, R. (2004). Difficultés d'apprentissage, processus de secondarisation et pratiques enseignantes : une hypothèse relationnelle. Revue Française de Pédagogie, 148, 89-100.

Benoist, J. (2003). À propos de l'éducation thérapeutique des patients. La place de l'anthropologie. Bulletin Amades [En ligne], 54 | 2003, mis en ligne le 01 juin 2004, consulté le 16 mars 2016. URL : http://amades.revues.org/716

Bril, B. (2002). Apprentissage et contexte. Intellectica, 35, 251-268.

Bureau, E. \& Hermann-Mesfen, J. (2014). Les patients contemporains face à la démocratie sanitaire. Anthropologie \& Santé [En ligne], 8 | 2014, mis en ligne le 21 avril 2015, consulté le 27 mars 2016. URL : http://anthropologiesante.revues.org/1342

Dewey, J. (1939). Creative democracy. In The Latter Works, Vol. 14. Carbondale : Southern Illinois University Press.

Debussche, X. (2015). L'observance en éducation thérapeutique : un concept pertinent ? Education, Santé, Sociétés, 1 (2), 213-227.

Fainzang, S. (2006). La relation médecins-malades : information et mensonge. Paris : PUF.

Fainzang, S. (2005). L'anthropologie : un outil au service de l'éducation pour la santé. La Santé de l'homme, 377.

Glaser, B.G. \& Strauss, A.L. (1967). The Discovery of Grounded Theory : Strategies for Qualitative Research. Hawthorne, NY : Aldine de Gruyter.

Greenhalgh, T. (2015). Health literacy : towards system level solutions. BMJ, 350 (February), h1026-. doi:10.1136/bmj.h1026

Lahire, B. (2005). L'invention de l'illettrisme. Rhétorique publique, éthique et stigmates. Paris : La Découverte.

Lahire, B. (2013). Dans les plis singuliers du social. Individus, institutions, socialisations. Paris : La Découverte.

Lenoir, Y., Larose, F., Deaudelin, C., Kalubi, J.-C. et Roy, G.-R. (2002). L'intervention éducative : clarifications conceptuelles et enjeux sociaux. Pour une reconceptualisation des pratiques d'intervention en enseignement et en formation à l'enseignement. Esprit critique, 4(4), 2-32. Leroux F. (1995). Démocratie et expérience : introduction à la démocratie créatrice de John Dewey. Horizons philosophiques, 5(2), 20-40.

Maingueneau, D. (2012). Que cherchent les analystes du discours? Argumentation et Analyse de discours. Mis en ligne le 15 octobre 2012, consulté le 15 novembre 2016. URL : http:// aad.revues.org/1354

Nutbeam, D. (2008). The evolving concept of health literacy. Social Science \& Medicine, 67(12), 2072-2078.

Nutbeam, D. (2000). Health literacy as a public health goal : a challenge for contemporary health education and communication strategies into the 21st century. Health Promotion International, 15 (3), 259-268.

Osborne, R.H., Batterham, R.W., Elsworth, G.R., Hawkins, M., Buchbinder, R. (2013). The grounded psychometric development and initial validation of the Health Literacy Questionnaire (HLQ). BMC Pub Health,13, 658. 
Rispail, M. (2011). Littéracie : une notion entre didactique et sociolinguistique - enjeux sociaux et scientifiques. En ligne (consulté le 15/11/2016) : http://www.forumlecture.ch/sysModules/ obxLeseforum/Artikel/446/2011_1_Rispail.pdf

Rothier-Bautzer, E. (2002). Pratiques soignantes en mutation : de la lutte contre la maladie à la collaboration avec le patient. Revue Française de pédagogie, 138, 39-50.

Stavo-Debauge, J. \& Trom, D. (2004). Le pragmatisme et son public à l'épreuve du terrain. Penser avec Dewey contre Dewey. In B. Karsenti \& L. Quéré (dir.) La croyance et l'enquête. Aux sources du pragmatisme. Raisons Pratiques, 15. Paris : Editions de l'EHESS.

Thille, P., Ward, N. \& Russell, G. (2014). Self-management education and support: Changing behaviors, or changing biomedical measures? Social Science \& Medicine,108, 97-105.

Thorne, S.E., Ternulf-Nyhlin, K. \& Paterson, B.L. (2000). Attitudes toward patient expertise in chronic illness. International journal of nursing studies, 37, 303-311.

\section{NOTES}

1. . International Diabetes Federation Diabetes. Atlas Sixth Edition: http://www.idf.org/ diabetesatlas (29/11/2014).

2. Ces entretiens ont été menés dans une perspective exploratoire qui visait à interroger nos modes de saisie et de traitement des données, afin de pouvoir présenter un travail longitudinal portant sur les deux années d'inclusion dans la recherche épidémiologique, ainsi que sur «l'après » recherche.

3. . Les situations d'apprentissage portent sur le risque cardiovasculaire, la maitrise des graisses dans l'alimentation, la gestion de l'activité physique ordinaire, les enjeux de la mise sous insuline, la prévention des complications des plaies du pied. Les supports utilisés sont ceux de la collection EPMC, présentée sur le site : www.litteratiesante.net

4. . Les discours sont présentés ici sous forme de tableau afin de mieux identifier les différences selon qu'ils relèvent des « niveaux » fonctionnel, interactif ou critique.

5. . International Diabetes Federation. IDF Diabetes Atlas, 6th edn. Brussels, Belgium: International Diabetes Federation, 2013. http://www.idf.org/diabetesatlas

6. . http://www.idf.org/diabetesatlas-new/how-to-cite

\section{RÉSUMÉS}

La contribution interroge les évolutions différenciées de 42 personnes malades chroniques inclues dans une recherche en 2012 et revues en 2015, à La Réunion. Le développement individuel et social est pensé en lien avec les interventions éducatives et de soins dont ont bénéficié ces 42 personnes durant la recherche. Les résultats montrent l'évolution de la littératie en santé à travers huit variables constitutives de la gestion du diabète de type 2 en contexte ordinaire. Ils montrent aussi que cette évolution n'est pas équivalente d'une variable à une autre. La discussion porte sur la pertinence d'envisager l'émancipation des personnes malades chroniques en lien avec la formation des soignants, ce qui invite à repenser la question de l'émancipation dans ses 
dimensions individuelles et sociales, en tenant compte des contextes pluriels dans lesquels elle s'actualise.

The present contribution analyzes the differentiated pathways of 42 persons with chronic illness, living in Reunion, who were included in a research in 2012 and re-interviewed in 2015. The individual and social development is considered within the framework of the educational interventions and care, which these 42 people benefited throughout the research period. The results show the progression of health literacy across eight constitutive variables of type 2 diabetes management in the ordinary context of the chronically ill person. They also show that trends are differentiated from one variable to another. The discussion focuses on the relevance to consider emancipation of chronically ill people in connection with the training of health professionals, and calls to rethink the question of emancipation in its individual and social dimensions, taking into account the plural contexts through which it develops.

\section{INDEX}

Keywords : empowerment, health literacy, patient education, training, chronic illness, type 2 diabetes, Reunion

Mots-clés : émancipation, littératie en santé, éducation thérapeutique, formation, malade chronique, diabète de type 2, La Réunion

\section{AUTEUR \\ MARYVETTE BALCOU-DEBUSSCHE}

Laboratoire Icare, EA 7389 - Université-ESPé de La Réunion 